\title{
Single Photon Thermal Ionization of $\mathbf{C}_{60}$
}

\author{
Klavs Hansen, ${ }^{1,2, *}$ Robert Richter, ${ }^{3}$ Michele Alagia, ${ }^{4}$ Stefano Stranges, ${ }^{4,5}$ Luca Schio, ${ }^{4}$ \\ Peter Salén, ${ }^{6}$ Vasyl Yatsyna, ${ }^{2}$ Raimund Feifel, ${ }^{2}$ and Vitali Zhaunerchyk ${ }^{2, \dagger}$ \\ ${ }^{1}$ Tianjin International Center of Nanoparticles and Nanosystems, Tianjin University, \\ 92 Weijin Road, Nankai District, Tianjin 300072, China \\ ${ }^{2}$ Department of Physics, University of Gothenburg, 41296 Gothenburg, Sweden \\ ${ }^{3}$ Elettra-Sincrotrone Trieste, Area Science Park, 34149 Basovizza, Trieste, Italy \\ ${ }^{4}$ IOM-CNR Tasc, SS-14, Km 163.5 Area Science Park, Basovizza 34149, Trieste, Italy \\ ${ }^{5}$ Dipartimento di Chimica e Tecnologie del Farmaco, Universitá Sapienza, Rome I-00185, Italy \\ ${ }^{6}$ Department of Physics, Stockholm University, 10691 Stockholm, Sweden
}

(Received 9 November 2016; revised manuscript received 4 January 2017; published 8 March 2017)

\begin{abstract}
We report on experiments which show that $\mathrm{C}_{60}$ can ionize in an indirect, quasithermal boiloff process after absorption of a single photon. The process involves a large number of incoherently excited valence electrons and yields electron spectra with a Boltzmann distribution with temperatures exceeding $10^{4} \mathrm{~K}$. It is expected to be present for other molecules and clusters with a comparatively large number of valence electrons. The astrophysical consequences are briefly discussed.
\end{abstract}

DOI: 10.1103/PhysRevLett.118.103001

The release of electrons upon irradiation of surfaces, as observed by Hertz [1], Lenard [2], and Hallwachs [3], gave an early impetus to the development of quantum mechanics with Einstein's explanation of the photoelectric effect [4]. Extending the photoelectric effect to electronic states with higher binding energies has permitted the mapping of the electronic structure and dynamics of atoms and molecules by means of photoelectron spectroscopy [5-7]. In the direct state-to-state ionization in these experiments, the energies of photoelectrons originating from a specific state increase with photon energy, reflecting that all energy in excess of the amount necessary to liberate the electrons is carried away by the particles as kinetic energy. The presence of a significant amount of low energy electrons in the few electronvolt $(\mathrm{eV})$ range that does not shift with photon energy indicates the presence of an ionization mechanism fundamentally different from this direct ionization.

We have observed such electrons in the ionization of $\mathrm{C}_{60}$ at photon energies between 27 and $65 \mathrm{eV}$. The experiments were performed at the GasPhase beam line of the Elettra synchrotron radiation facility [8]. The electrons were detected with a velocity map imaging (VMI) spectrometer [9]. An ion time-of-flight mass spectrometer was mounted back-to-back with the VMI [10]. This allowed us to selectively measure electrons originating from single ionization of $\mathrm{C}_{60}$, in coincidence with the $\mathrm{C}_{60}^{+}$ parent ion or fragmentation products, primarily $\mathrm{C}_{58}^{+}$and $\mathrm{C}_{56}^{+}$. To efficiently eliminate detection of accidental coincidences, the event count rate was kept more than $10^{4}$ times lower than the repetition rate of the synchrotron pulses. The kinetic energy scale was calibrated by recording photoelectron spectra of helium with varying photon energy. The measured electron kinetic energy resolution was $6.5 \%$ at $15 \mathrm{eV}$ kinetic energy. The synchrotron radiation was polarized parallel to the detector plane, which allows a reconstruction of the original three-dimensional velocity distribution from the measured two-dimensional data $[11,12]$. The molecules originated from an effusive oven kept at $480^{\circ} \mathrm{C}$ and set $15 \mathrm{~cm}$ from the ionization region. The pressure in the ionization chamber was $3 \times 10^{-8}$ mbar. The photon flux was recorded using a photodiode placed at the end of the beam line. To minimize the contribution of higher order light from the beam line, solid thin foil ( $\mathrm{Sn}, \mathrm{Mg}$, and $\mathrm{Al}$ ) and gas (neon) filters were inserted into the light path.

The raw VMI images of electron velocities measured in coincidence with $\mathrm{C}_{60}^{+}$ions at the photon energies of $17 \mathrm{eV}$, $19 \mathrm{eV}, 29 \mathrm{eV}$, and $31 \mathrm{eV}$ are shown in Fig. 1. The tendency for photoelectrons to appear at low energy with increasing photon energy is clearly visible already in these unprocessed distributions. The 17 and $19 \mathrm{eV}$ VMI images [Figs. 1(a) and 1(b)] also show two rings associated with the direct photoionizations from the two highest occupied molecular orbitals (HOMO and HOMO-1), with radii that grow with photon energy. In contrast, the high-intensity central peak does not move toward higher energy with increasing photon energy, excluding an origin from direct ionization of a molecular orbital below these two states.

Ionization from single molecular orbitals does not produce these spectra. The lowest $2 a_{g}$ molecular orbital has the energy $27.5 \mathrm{eV}$ below vacuum [13], and should appear in the electron spectra at 29 and $31 \mathrm{eV}$ photon energies [Figs. 1(c) and 1(d)]. Instead, low-energy electrons are seen at both higher and lower photon energies (Fig. 1). Also, low energy electrons produced in potential double ionization processes are excluded by the applied coincidence technique and, below the threshold of $19.0 \mathrm{eV}$ [14], even by energy conservation. The presence of such low-energy electrons 
(a) $h v=17 \mathrm{eV}$
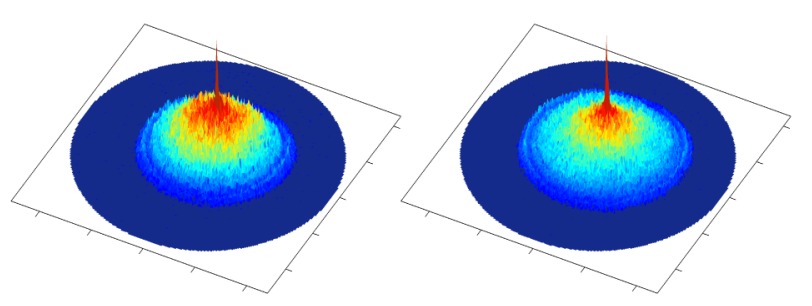

(c) $h v=29 \mathrm{eV}$
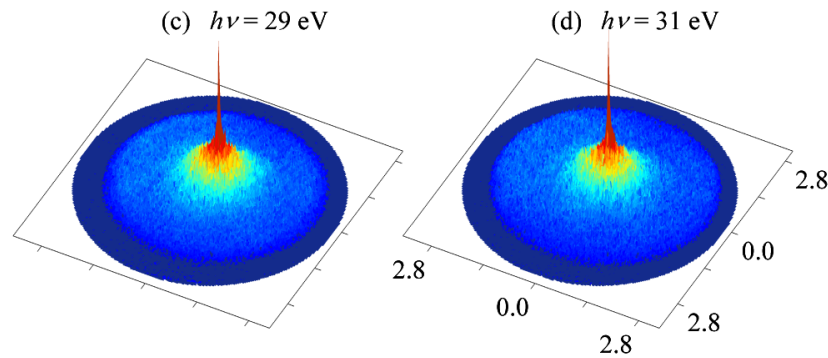

FIG. 1. Raw VMI transverse velocity distributions measured in coincidence with $\mathrm{C}_{60}^{+}$at the photon energies given. The labels on the axes of panel (d) give the electron speeds parallel to the detector surface in units of $10^{6} \mathrm{~m} / \mathrm{s}$. The images are normalized to identical total intensities.

implies that the major part of the maximum excess energy, $h \nu-\Phi$ (the ionization energy, $\Phi$, is $7.6 \mathrm{eV}[15]$ ), remains in the ion, in striking contrast to direct ionization.

Another important difference between electrons generated by direct ionization and the low energy electrons observed here is their angular distribution. This is illustrated in Fig. 2, which shows the VMI image recorded at $h \nu=17 \mathrm{eV}$. Figures 2(b)-2(d) present angular resolved plots of intensities for different radial distances of the image, as indicated by the rings which correspond to the kinetic energies of $\approx 8.0$ and $9.4 \mathrm{eV}$. The intensities have a clear angular variation [Figs. 2(c) and 2(d)], in strong contrasts to the low energy part, which has no discernible angular variation, corresponding to an asymmetry parameter $\beta$ of zero. The value $\beta=0$ can also occur for individual quantum states. However, the low kinetic energy angular distributions observed here are consistently symmetric, showing no correlations with the angle to the light polarization. This indicates a very efficient decoupling of the electron emission from the initial excitation process, and corroborates our assertion that emission of the low kinetic energy electrons is not direct and that the ionization mechanism producing these electrons is fundamentally different from direct photoionization.

The deconvoluted and angle-averaged electron spectra, measured in coincidence with $\mathrm{C}_{60}^{+}$, are shown in Figs. 3(a)3(h) for a series of photon energies from 13.5 to $60.0 \mathrm{eV}$. The (mass-filtered) electron images were inverted using the Windows implementation of the PBASEX software [11] and the maximum entropy method [12]. The amount of low kinetic energy electrons is observed to increase with photon energy, and the peak takes a shape recognizable as
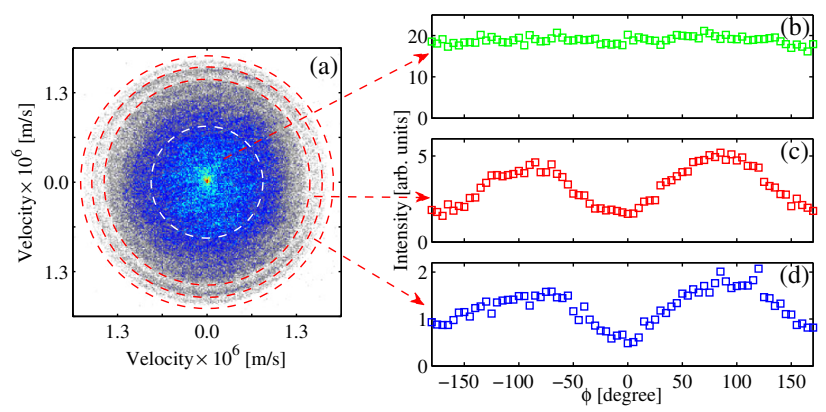

FIG. 2. (a) VMI image recorded at the photon energy $h \nu=17 \mathrm{eV}$. (b)-(d) show electron counts versus different polar angles for low kinetic energy electrons (b) and for electrons emitted upon direct ionization of HOMO-1 (c) and HOMO (d).

exponential. In contrast to direct ionization, thermal emission of charged particles in a Coulomb potential gives rise to near-exponential distributions [16] with the product temperature as the characteristic energy [17], and we take the exponentials observed in the present experiments as evidence for a thermal emission process.

As an important side remark, we note that, although the ionization yield associated with the surface plasmon resonance [21] is effectively continuous and will, therefore, give contributions at low electron energies for all photon energies in the range studied, absorption into this resonance is not responsible for the phenomena observed here. The slope of the low electron energy distributions predicted from a simple application of the orthodox theory (i.e., the photoelectric effect theory) is contrary to our experimental observations at the photon energies above the plasmon peak energy of $\approx 22 \mathrm{eV}$. Therefore, an understanding of the electron spectra as a simple mapping of the plasmon profile is ruled out.

The process observed here is also not conventional thermionic emission, which is the statistical electron emission from a completely thermalized molecule. The excitation energy required for such a process for $\mathrm{C}_{60}$ is twenty eV above the $23-27 \mathrm{eV}$ onset energy seen in Fig. 3 of Ref. [22]. The thermal-like electron emission observed in these experiments must, therefore, proceed through excitation and equilibration of a small subset of all possible degrees of freedom. The electronic degrees of freedom provide this gateway because electrons carry the largest part of the dipole oscillator strength and, at the same time, have a small heat capacity, permitting a thermal emission reaction for only a fraction of the excitation energy required if vibrational degrees of freedom were also excited. The large difference in the number of possible states that can be populated with purely electronic or purely vibrational excitations is demonstrated with the level densities of the two subsystems, which at the typical excitation energy $30 \mathrm{eV}$, take the values $10^{15} \mathrm{eV}^{-1}$ and $10^{142} \mathrm{eV}^{-1}$ for electronic and vibrational excitations, respectively $[16,23]$. Therefore, the electrons are likely to be emitted 


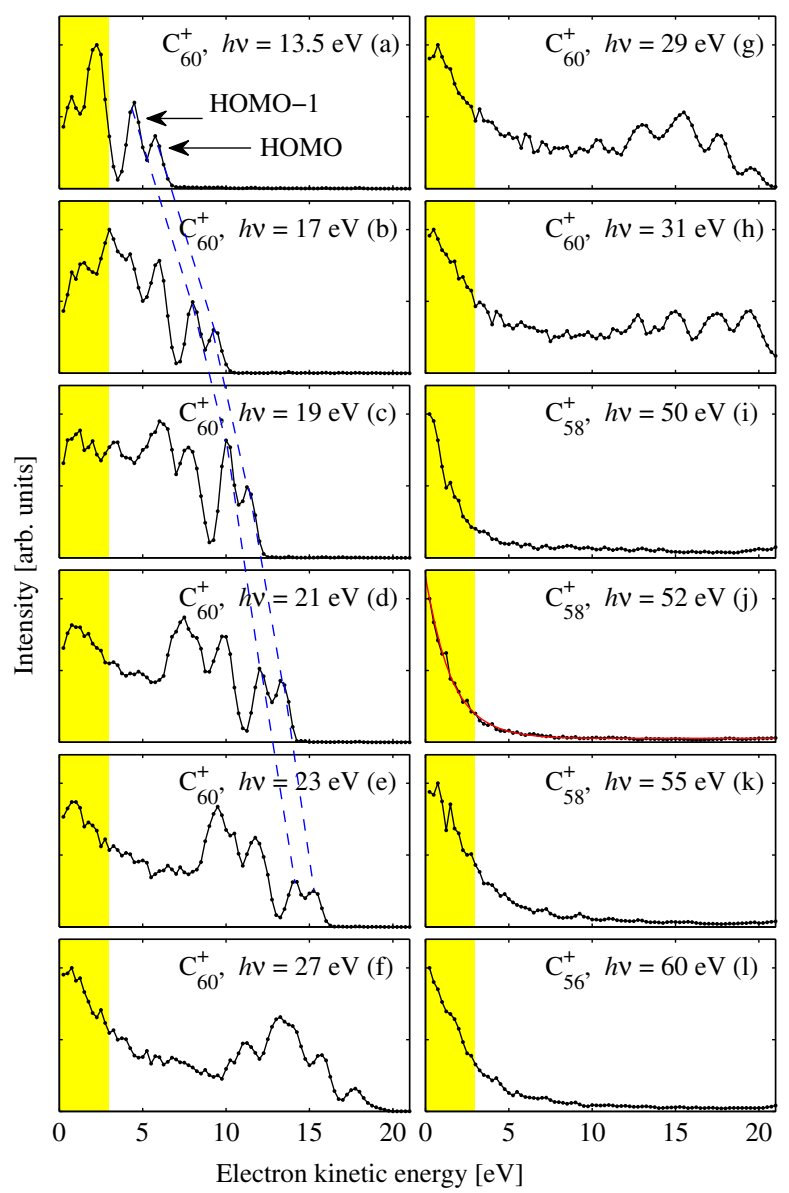

FIG. 3. Electron spectra deconvoluted from the VMI images measured in coincidence with $\mathrm{C}_{60}^{+}(\mathrm{a})-(\mathrm{h}), \mathrm{C}_{58}^{+}(\mathrm{i})-(\mathrm{k})$, and $\mathrm{C}_{56}^{+}$ (l) at several different photon energies. The dashed blue curves depict shifts of the HOMO and HOMO-1 with the photon energy. The electron spectra show that the relative intensities of HOMO and HOMO-1 vary as a function of photon energy, consistent with the observations made in [18-20]. The yellow areas highlight the low kinetic energy range $\left(E_{k} \leq 3 \mathrm{eV}\right)$. The red curve in (j) is $10^{-3}+0.04 \exp \left(-E_{k} / k T\right)$ with $k T=1.58 \mathrm{eV}$.

from incoherently excited electronic motion. In this connection, the vibrational degrees of freedom act only as an inactive continuum of states into which the electronic excitations are dissipated. The mechanism is illustrated in Fig. 4. After the initial equilibration and before the excess energy is distributed over vibrational modes, the incoherently excited, internally thermalized electrons may emit an electron in a thermal process [16]. This channel is open during a period of a few hundred femtoseconds, after which the electrons are equilibrated with the vibrational degrees of freedom. Complete equilibration is reached after a picosecond or less $[16,24,25]$. The ionization mechanism outlined here may also have bearings on the interpretation of the collision-induced ionization experiments reported in Ref. [26], where the ionization threshold of $\mathrm{C}_{70}^{-}$was shifted up from the ionization energy with an amount similar to that observed here for $\mathrm{C}_{60}$.

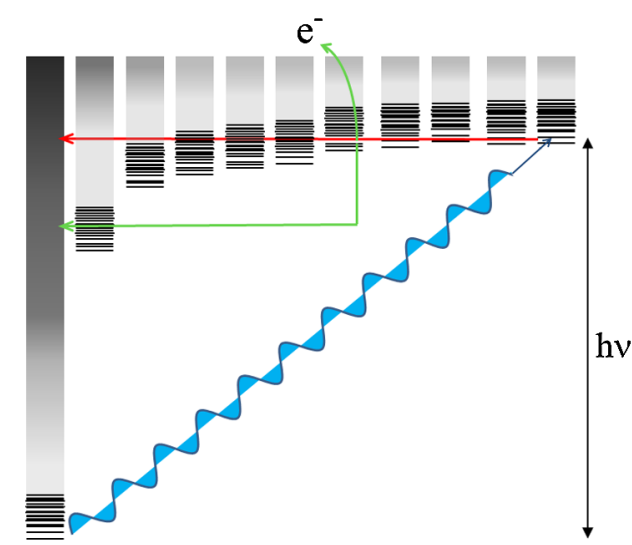

FIG. 4. The ionization process in a schematic, multilevel Jablonski diagram. Photoexcitation promotes the molecule to a highly excited electronic state, with the vibrational degrees of freedom (indicated as lines and grey areas) as spectators. During a time on the order of several hundred femtoseconds, the molecule moves across a number of states with increasingly lower electronic ground state energies and correspondingly higher vibrational excitation energy (red arrow). The motion should be understood as an average drift. During this drift, an electron may be emitted in a stochastic process (green arrow).

A theoretical framework for a description of this initial equilibration process is outlined in Refs. [27,28] and in the context of nuclear physics statistical reactions in Ref. [29]. The ionization mechanism has strong analogies to nuclear decay after neutron absorption, the different excitation mechanisms and energy scales notwithstanding. The salient points for those systems are summarized in the compound nucleus concept [17,29], which builds on efficient dissipation of energy among the fermionic constituents of a nucleus. A hint of the detailed mechanism of the initial excitation and relaxation is found in the fact that the low energy electrons also appear for photon energies that reach below the energy of the lowest molecular orbital, but are still far above core level energies. This demonstrates that the initial process must involve at least two particle-two hole excitations and possibly higher orders.

Transient hot electron ionization has been observed previously for multiphoton ionization of large molecules with femtosecond pulses of photons with energies far below the ionization energy [30-32] and in multiphoton ionization of large rare gas clusters with vacuum ultraviolet photons [33]. A crucial difference is that, in the present experiments, the electrons are emitted after absorption of a single, comparatively high energy photon. Thus, it will appear much more widespread in nature, e.g., in astrophysical settings, than the processes requiring multiphoton absorption in femtosecond laser pulses generated in the artificial environment of a laser laboratory.

The energy left in the ion after the thermal-like ionization process is available for subsequent molecular fragmentation on the much longer time scale, a fraction of a microsecond, set by the extraction time of the ion mass spectrometer. As for 


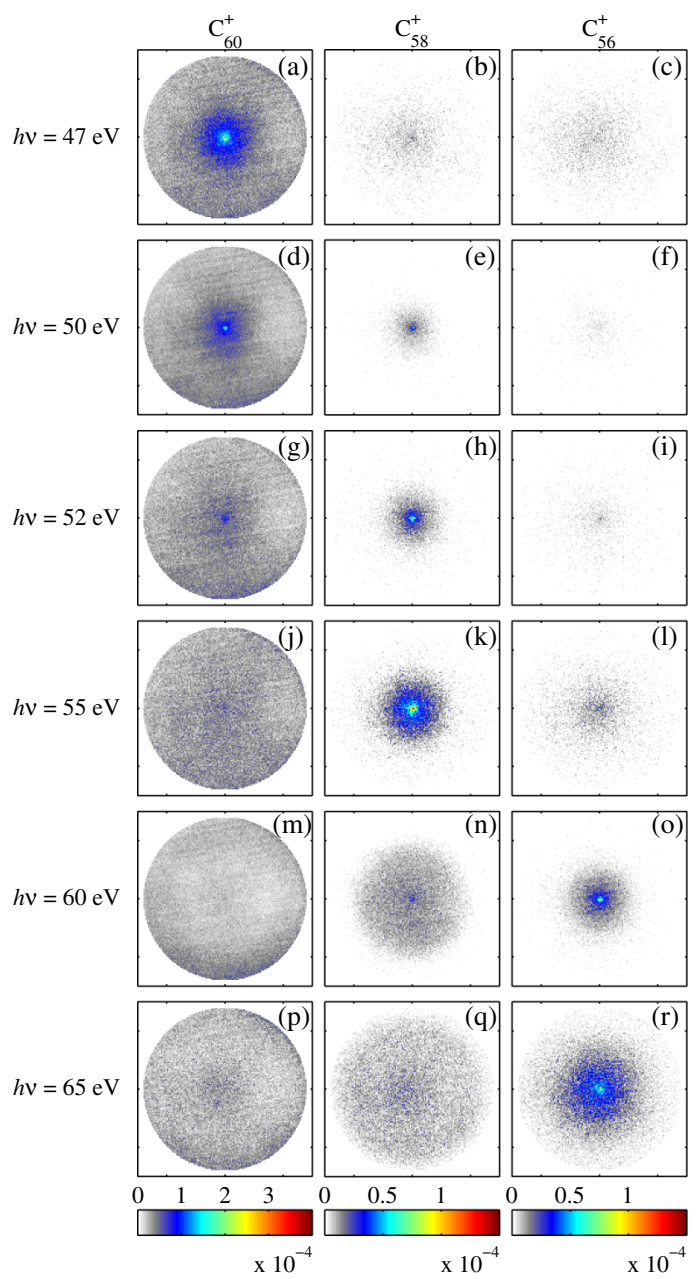

FIG. 5. VMI spectra measured in coincidence with $\mathrm{C}_{60}^{+}, \mathrm{C}_{58}^{+}$, and $\mathrm{C}_{56}^{+}$, at the photon energies indicated. The photon energies at which the frames are recorded are given to the left, and the ions measured in coincidence are given at the top.

thermionic emission, this fragmentation happens at internal energies that are significantly higher than the threshold energies, in this case the sum of the ionization and dissociation energies of $18.4 \mathrm{eV}[34,35]$. $\mathrm{C}_{60}$-electron impact and photofragmentation experiments $[22,36]$ locate the onset of $\mathrm{C}_{60}^{+} \rightarrow \mathrm{C}_{58}^{+}$around 50 and $56-58 \mathrm{eV}$ for the further fragmentation of $\mathrm{C}_{58}^{+}$. Figure 5 shows the development of the spectra as a function of photon energy for electrons in coincidence with $\mathrm{C}_{N}^{+}(N=60,58,56)$. The shift in intensity in the coincidence spectra in Fig. 5 conforms very closely to the photon energies previously observed [36], with the low energy electrons only appearing in coincidence with $\mathrm{C}_{60}^{+}$up to photon energies slightly above $50 \mathrm{eV}$, after which they disappear and reappear in coincidence measurements with $\mathrm{C}_{58}^{+}$at approximately $h \nu=52-55 \mathrm{eV}$. Between $h \nu=55$ and $60 \mathrm{eV}$, this signal then switches off and electrons appear, instead, in coincidence with $\mathrm{C}_{56}^{+}$. The fact that a fragment ion appears only above about $50 \mathrm{eV}$ and not around the $18.4 \mathrm{eV}$ threshold excludes direct electron promotion to antibonding orbitals upon excitation. Thermionic emission of a fragmentation product is also ruled out, because the electron spectra generated in this process would be characterized by a significantly lower temperature $(0.3 \mathrm{eV})$ [37] compared with the electron temperature of $\approx 1.6 \mathrm{eV}$ [Fig. 3(j)] observed here.

The novel ionization mechanism described here will have fundamental repercussions for our understanding of the ionization of matter. It also provides a hitherto unconsidered source of ionization and low energy electrons in the harsh environment of the dense molecular clouds in the star-forming regions of space. $\mathrm{C}_{60}^{+}$, for example, is known to exist in the diffuse interstellar clouds $[38,39]$. The attachment of the low energy electrons produced in the ionization to neutral molecules forming negative ions, as recently discovered [40], will be very efficient because the cross section varies as $E^{-1}$ at low collision energy [41].

This work has been supported by the Swedish Research Council (VR) and the Knut and Alice Wallenberg Foundation, Sweden. Financial support by the Italian Istituto Officina dei Materiali (IOM-CNR) through the Free Electron Lasers of Europe (EUROFEL) Project is also acknowledged (L.S.). The authors also acknowledge the open access contribution of the Research Infrastructure (RI) Elettra. The research leading to these results has received funding from the European Community's Seventh Framework Programme (FP7/2007-2013) under Grant No. 312284.

*KlavsHansen@tju.edu.cn

†vitali.zhaunerchyk@physics.gu.se

[1] H. Hertz, Ann. Phys. (Berlin) 267, 983 (1887).

[2] P. Lenard, Ann. Phys. (Berlin) 313, 149 (1902).

[3] W. L. F. Hallwachs, Ann. Phys. Chem. 33, 301 (1888).

[4] A. Einstein, Ann. Phys. (Berlin) 322, 132 (1905).

[5] K. Siegbahn et al., ESCA Applied to Free Molecules (North-Holland, Amsterdam, 1969).

[6] H. Niikura, F. Légaré, R. Hasbani, M. Yu Ivanov, D. M. Villeneuve, and P. B. Corkum, Nature (London) 421, 826 (2003).

[7] P. Johnsson et al., Phys. Rev. Lett. 95, 013001 (2005).

[8] R. R. Blyth et al., J. Electron Spectrosc. Relat. Phenom. 101-103, 959 (1999).

[9] A. T. J. B. Eppink and D. H. Parker, Rev. Sci. Instrum. 68, 3477 (1997).

[10] P. O'Keeffe et al., Rev. Sci. Instrum. 82, 033109 (2011).

[11] G. A. Garcia, L. Nahon, and I. Powis, Rev. Sci. Instrum. 75, 4989 (2004).

[12] B. Dick, Phys. Chem. Chem. Phys. 16, 570 (2014).

[13] P. Colavita, G. De Alti, G. Fronzoni, M. Stener, and P. Decleva, Phys. Chem. Chem. Phys. 3, 4481 (2001).

[14] A. V. Pogulay, R. R. Abzalimov, S. K. Nasibullaev, A. S. Lobach, T. Drewello, and Y. V. Vasil'ev, Int. J. Mass Spectrom. 233, 165 (2004).

[15] D. L. Lichtenberger, K. W. Nebesny, C. D. Ray, D. R. Huffman, and L. D. Lamb, Chem. Phys. Lett. 176, 203 (1991). 
[16] K. Hansen, K. Hoffmann, and E. E. B Campbell, J. Chem. Phys. 119, 2513 (2003).

[17] V. Weisskopf, Phys. Rev. 52, 295 (1937).

[18] T. Liebsch, O. Plotzke, F. Heiser, U. Hergenhahn, O. Hemmers, R. Wehlitz, J. Viefhaus, B. Langer, S. B. Whitfield, and U. Becker, Phys. Rev. A 52, 457 (1995).

[19] S. Korica, D. Rolles, A. Reinköster, B. Langer, J. Viefhaus, S. Cvejanovi, and U. Becker, Phys. Rev. A 71, 013203 (2005).

[20] A. Rüdel, R. Hentges, U. Becker, H. S. Chakraborty, M. E. Madjet, and J. M. Rost, Phys. Rev. Lett. 89, 125503 (2002).

[21] I. V. Hertel, H. Steger, J. de Vries, B. Weisser, C. Menzel, B. Kamke, and W. Kamke, Phys. Rev. Lett. 68, 784 (1992).

[22] M. Foltin, M. Lezius, P. Scheier, and T. D. Märk, J. Chem. Phys. 98, 9624 (1993).

[23] T.Beyer and D. F. Swinehart, Commun. ACM 16, 379 (1973).

[24] J.-Y. Bigot, J.-C. Merle, O. Cregut, and A. Daunois, Phys. Rev. Lett. 75, 4702 (1995).

[25] C. Voisin, D. Christofilos, N. Del Fatti, F. Vallée, B. Prével, E. Cottancin, J. Lermé, M. Pellarin, and M. Broyer, Phys. Rev. Lett. 85, 2200 (2000).

[26] P. Scheier, D. Hathiramani, W. Arnold, K. Huber, and E. Salzborn, Phys. Rev. Lett. 84, 55 (2000).

[27] N. Slama, P.-G. Reinhard, and E. Suraud, Ann. Phys. (Amsterdam) 355, 182 (2015).

[28] J. R. M. Saavedra, A. Asenjo-Garcia, and F. J. Garcia de Abajo, ACS Photonics 3, 1637 (2016).
[29] A. J. Cole, in Statistical Models for Nuclear Decay, (Institute of Physics Publishing, Philadelphia, 2000), p. 144.

[30] E. E. B. Campbell, K. Hansen, K. Hoffmann, G. Korn, M. Tchaplyguine, M. Wittmann, and I. V. Hertel, Phys. Rev. Lett. 84, 2128 (2000).

[31] N. Pontius, G. Lüttgens, P. S. Bechthold, M. Neeb, and W. Eberhardt, J. Chem. Phys. 115, 10479 (2001).

[32] M. Kjellberg, O. Johansson, F. Jonsson, A. V. Bulgakov, C. Bordas, E. E. B. Campbell, and K. Hansen, Phys. Rev. A 81, 023202 (2010).

[33] T. Laarmann, M. Rusek, H. Wabnitz, J. Schulz, A. R. B. de Castro, P. Gürtler, W. Laasch, and T. Möller, Phys. Rev. Lett. 95, 063402 (2005).

[34] R. K. Yoo, B. Rustic, and J. Berkowitz, J. Chem. Phys. 96, 911 (1992).

[35] S. Tomita, J. U. Andersen, K. Hansen, and P. Hvelplund, Chem. Phys. Lett. 382, 120 (2003).

[36] A. Reinköster, S Korica, G Prümper, J Viefhaus, K Godehusen, O Schwarzkopf, M Mast, and U Becker, J. Phys. B 37, 2135 (2004).

[37] F. Lépine and C. Bordas, Phys. Rev. A 69, 053201 (2004).

[38] E. K. Campbell, M. Holz, D. Gerlich, and J. P. Maier, Nature (London) 523, 322 (2015).

[39] J. P. Maier and E. K. Campbell, Phil. Trans. R. Soc. A 374, 20150316 (2016).

[40] M. C. McCarthy, C. A. Gottlieb, H. Gupta, and P. Thaddeus, Astrophys. J. Lett. 652, L141 (2006).

[41] E. P. Wigner, Phys. Rev. 73, 1002 (1948). 